\title{
Evolving role of cardiovascular pathology in clinical and surgical decision making
}

Gaetano Thiene, MD, FRCP*

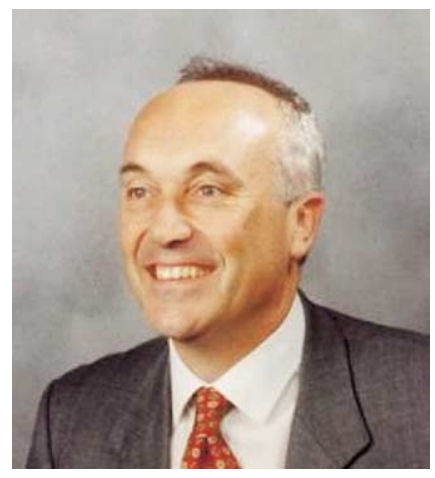

Dr Thiene
From the Department of Pathology, University of Padua Medical School, Padua, Italy. Received for publication July 14, 2004; accepted for publication July 30, 2004.

Address for reprints: Gaetano Thiene, MD, FRCP, Istituto di Anatomia Patologica, Università degli Studi di Padova, Via A. Gabelli, 61, 35121 Padova, Italy (E-mail: gaetano.thiene@unipd.it).

*Professor G. Thiene is President of the Society for Cardiovascular Pathology, 2003-2005.

J Thorac Cardiovasc Surg 2005;129:966-9

$0022-5223 / \$ 30.00$

Copyright $\odot 2005$ by The American Association for Thoracic Surgery

doi:10.1016/j.jtcvs.2004.07.063
$\mathrm{W}$ hen I was still a student in the late 1960s and started following the call for cardiovascular pathology, the job was restricted mostly to autopsy. This discipline was the Cinderella within the large family of anatomic pathology, and the reason was that it dealt only with cadavers.

Cardiac surgery was just starting, and there was high operative mortality. The cardiovascular pathologist was asked to perform autopsies on surgical fatalities, bearing witness to limitations in medical knowledge. Cardiopulmonary bypass and cardiac arrest allowed the surgeons to open the heart and correct the defects, being still unaware of elementary morphologic notions, such as the topography of the conduction system. Most of the septal defects first operated on at the Mayo Clinic with the use of a cardiopulmonary bypass machine were weaned off the pump with atrioventricular block. ${ }^{1}$

Operable cardiac diseases consist of gross structural defects, and clearly the mission of the pathologist was to support the clinicians and surgeons by transferring the postmortem information into knowledge of clinical and surgical anatomy to improve the diagnosis and treatment for the benefit of the patient. The anatomic theater was still the place mors ubi gaudet succurrere vitae (where death enjoys to help life). ${ }^{2}$

It soon became evident that the cardiologist, the cardiac surgeon, and the cardiovascular pathologist should possess a similar cultural background, despite different professional duties. The catheterization laboratory, the surgical theater, and the autopsy room were places where the same teaching and language for fruitful clinicopathologic correlations were applied. ${ }^{3}$

After improvements in anesthesiology and myocardial-cerebral protection during cardiac arrest, which rendered operations feasible in neonates, ${ }^{4}$ a sound knowledge of the anatomy of complex congenital heart disease appeared to be a prerequisite for optimizing clinical diagnosis and improving surgical repair. Implementation of a cardiac registry of congenital heart disease specimens allowed the study of the whole anatomic spectrum of various defects. The segmental approach (atria, ventricles, and great arteries) was introduced as the best way to clear up the difficulty of diagnosing complex congenital heart diseases, with the aim of reconstruction by means of operatively separated pulmonary and systemic circulations in a right sequential manner. ${ }^{5}$ The identification of the precise course of the conduction system through histologic serial sections (Figure 1) virtually removed all the risks of atrioventricular block during closure of septal defects by guiding the hand of the surgeon in suturing the patch.

The early occurrence of pulmonary vascular disease was found to jeopardize the surgical repair of congenital heart disease with septal defects (Figure 2) and to suggest the need to plan the time of operation within the first year of life. It is quite rewarding to realize that thanks to the improvement of diagnostic and surgical expertise with the help of the pathologist, the mortality for repair of congenital heart disease, including quite complex forms, such as univentricular hearts, decreased drastically with time.

Any tissue resected during cardiac surgery started undergoing routine histologic analysis, thus opening the era of cardiovascular surgical pathology. Valves, vessels, venous or arterial grafts, pericardium, and tumors were submitted systematically to a precise microscopic diagnosis, an essential step in establishing the nosography and epidemiology of operable cardiovascular disease (Figure 3). 
The increasing use of valvular prostheses, with an intrinsic propensity toward structural failure, required a novel skill. Bioprosthetic valves were found to have limited durability, with a half-life of only 8 to 10 years. Calcification proved to be the main cause of failure, with cusp stiffening, disruption, or both. ${ }^{6}$ In vivo animal studies and in vitro experiments were carried out to test antimineralization agents, with the aim of introducing new devices with a 5- to 7-year increase of half-life in clinical practice. ${ }^{7}$

In the past there was a common quote, "The pathologist knows everything, but too late." This limitation changed abruptly in the cardiovascular field with the introduction of the endomyocardial biopsy. ${ }^{8}$ Endomyocardial biopsy is today the gold standard for diagnosis of myocarditis, giving the cardiovascular pathologist the final word.

The pivotal role of the cardiovascular pathologist was further enhanced by the beginning of a cardiac transplantation program, which requires the use of endomyocardial biopsy for rejection monitoring. The study of heart specimens resected at the time of transplantation resulted in an extraordinary opportunity for research with the discovery of new morbid entities, such as requiring a new World Health Organization classification of cardiomyopathies. ${ }^{9}$

On the autopsy side, a new demand for cardiovascular pathology came from the amazing occurrence of sudden death, even in young people or athletes collapsing on the playground. The concept that the heart might stop suddenly because of ventricular fibrillation in the absence of an ischemic milieu was a revolutionary step forward for the pathologist. We soon realized that the working ventricular myocardium itself might be the substrate of life-threatening arrhythmias, despite the integrity of the conduction system and the coronary arteries. Arrhythmogenic right ventricular cardiomyopathy, which was discovered at autopsy to be the major cause of sudden death in the young, ${ }^{10}$ represented an alarming instance insofar as fibrofatty replacement of the right ventricular free wall might trigger lethal tachyarrhythmias during effort through reentrant mechanisms, even in the setting of a normal left ventricle and conduction system.

The traditional tools of the pathologist are the eyes at autopsy for gross dissection and the light microscope for histologic examination. Magnification power increased significantly with electron microscopy, allowing morphologic investigation at the subcellular level. However, there are offending organisms or structural defects that escape ultrastructural imaging, emphasizing the limits of traditional morphology. The discovery of new molecular biology tools, such as in situ hybridization and the polymerase chain reaction (PCR), as well as gene cloning and sequencing, opened extraordinary avenues for new insight deep in the cell and nuclear pathophysiology, as well as in the genes and coded proteins. The collaboration with clinicians was

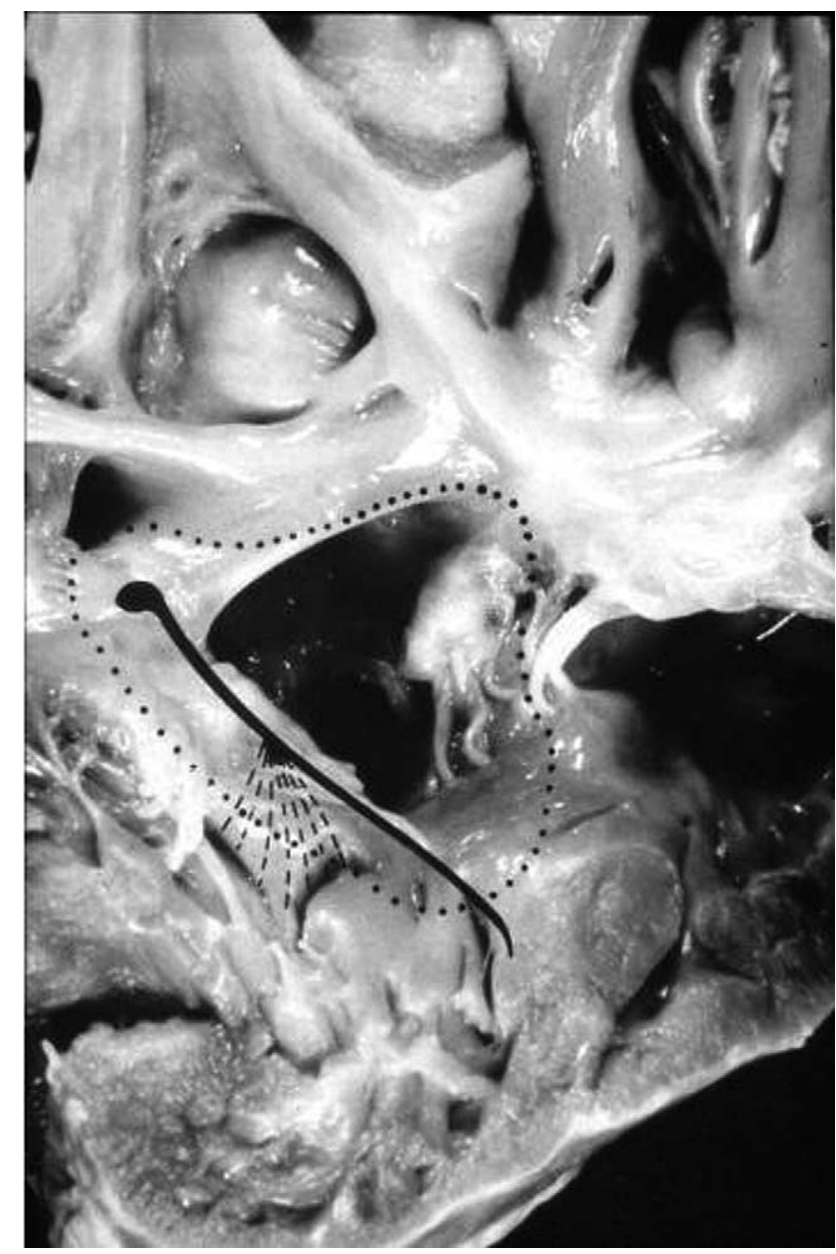

Figure 1. The course of atrioventricular conduction tissue in complete atrioventricular canal. The dotted line indicates how to insert the surgical patch so as to avoid atrioventricular block.

extended to basic scientists, and this policy has been rewarding insofar as in 15 years it has been possible to move from the first postmortem observation of familiar arrhythmogenic right ventricular cardiomyopathy to the discovery of the culprit gene. ${ }^{11}$

The use of PCR or reverse transcriptase PCR, able to amplify DNA or RNA by up to 1 billion copies, was a powerful weapon in detecting viral particles, which were found in up to $50 \%$ of endomyocardial biopsy specimens from patients with myocarditis. DNA viruses, like adenovirus, were found to be cardiotropic as much as RNA enterovirus. PCR is now an essential tool in endomyocardial biopsy evaluation to achieve a gold standard for diagnosis.

Up to $10 \%$ of young people dying suddenly present with a normal heart (mors sine materia). For the pathologist, it was frustrating and difficult to accept that patients who die suddenly had a normal heart, even after extensive morpho- 

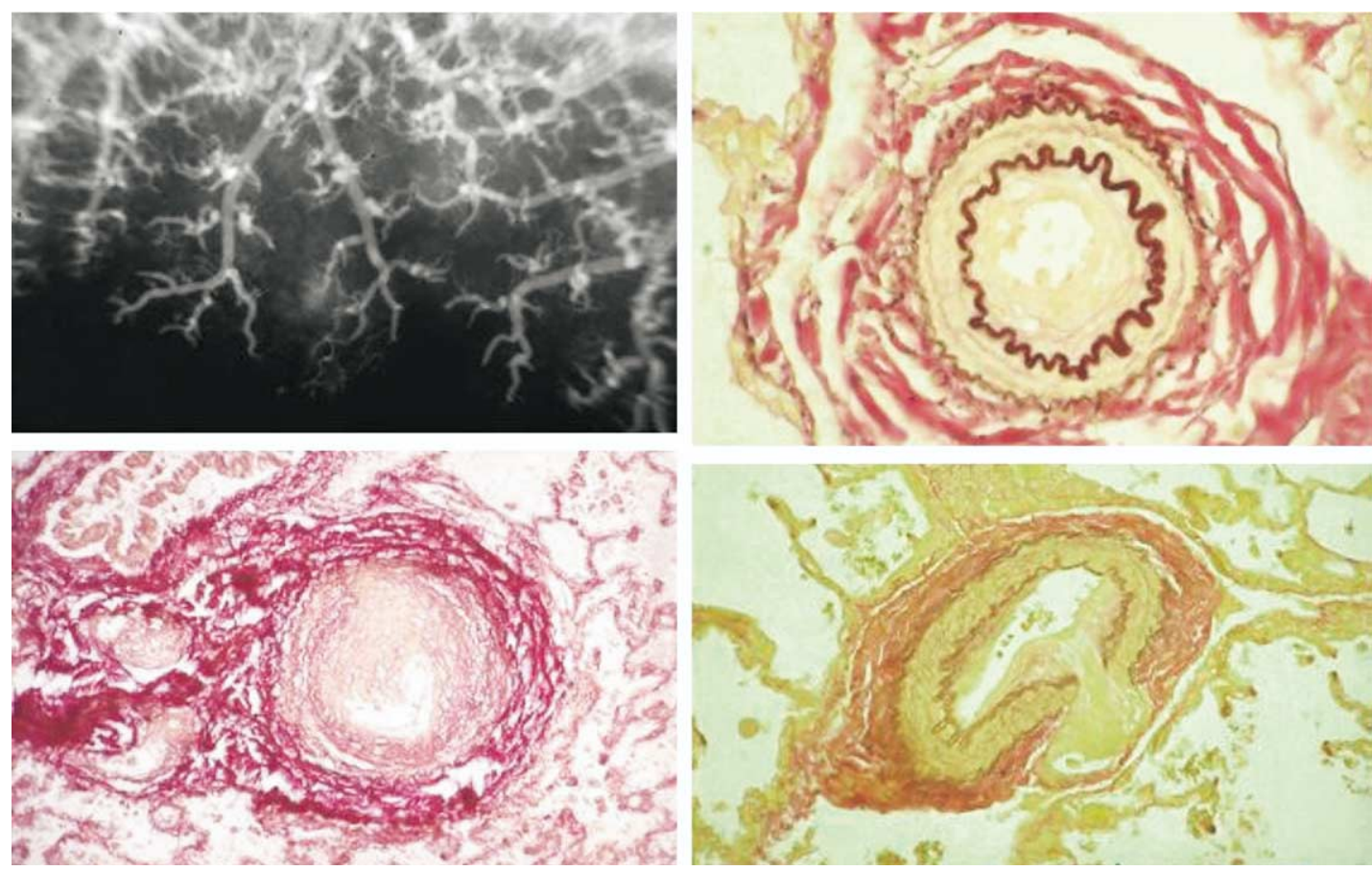

Figure 2. Pulmonary vascular disease in congenital heart defects with a left-to-right shunt. Note the winter-tree feature of the pulmonary artery on the postmortem angiogram.

logic examination including the conduction system. Molecular genetics demonstrated that many of these cases are ascribable to genes encoding defective proteins, receptors, or both of cell-sarcoplasmic reticulum membranes, where ion channels are located. Clearly, these defects are beyond the magnification power of any microscope. A mutation of a single base might account for an altered coded protein with only one different amino acid (Figure 4), enough to impair the receptor function and $\mathrm{Ca}^{++}$release, jeopardizing the electrical stability during effort. ${ }^{11}$ One might wonder whether this infinitesimal gene sequence change might be enough to deserve the name of congenital heart disease, namely cardiac structural defect present at birth. Most probably, with the advent of molecular genetics, the definition of congenital disease will change to include not only structural but also gene defects present at birth.

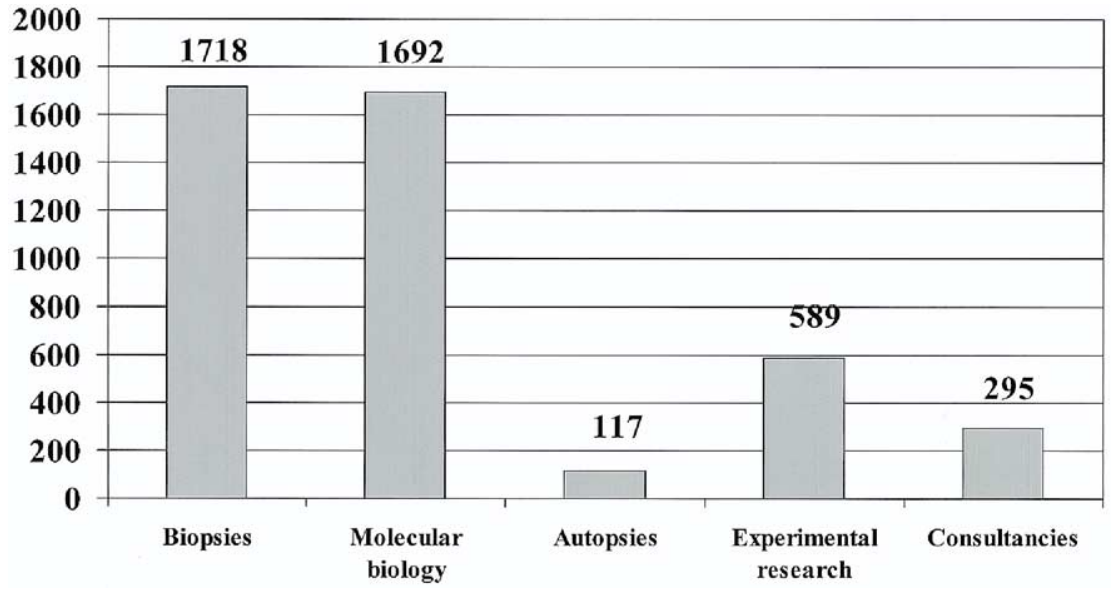

Figure 3. Number and types of reports at the Cardiovascular Pathology Service, University of Padua, 2001 (number of reports, 3309). 


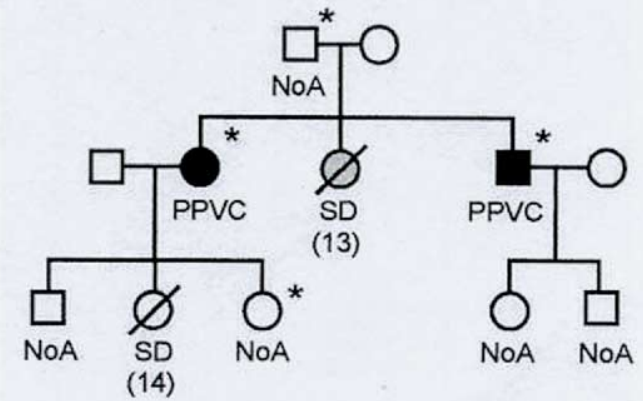

Family 126

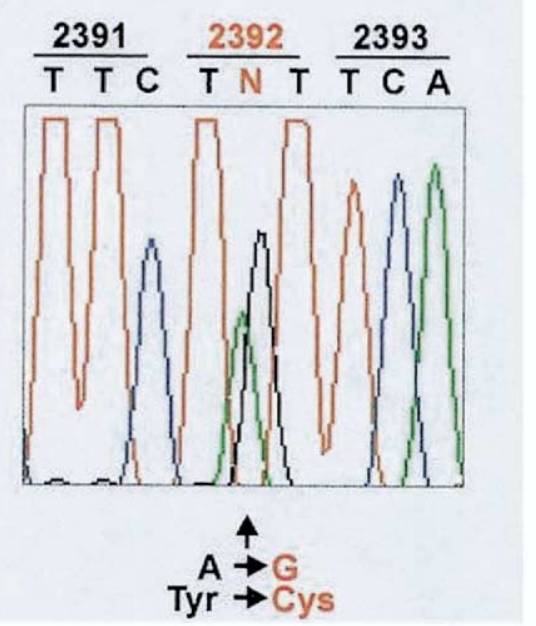

Figure 4. Pinpoint mutation of ryanodine receptor 2 in a family with sudden death. Mutation of the base adenine into guanine accounted for amino acid substitution from tyrosine into cysteine. NoA, No arrhythmias; PPVC, polymorphous premature ventricular complex; SD, sudden death; open symbols, unaffected; filled symbols, affected; gray symbols, unknown. *Mutation carrier. (Reprinted from the Journal of the American College of Cardiology, vol 40, Bauce B, Rampazzo A, Basso C, Bagattin A, Daliento L, Tiso N, et al. Screening for Ryanodine Receptor Type 2 Mutations in Families With Effort-Induced Polymorphic Ventricular Arrhythmias and Sudden Death: Early Diagnosis of Asymptomatic Carriers. p. 341-9, (C) 2002, with permission from The American College of Cardiology Foundation.)

Death is an unavoidable outcome of human life, and there will always be a need for autopsy, whether clinical or forensic. The anatomy theater will continue to be an essential laboratory of cardiovascular pathology. However, it is becoming more and more evident that the ability to interpret the morphologic alterations should be based on a solid clinical and biologic background. The pathologist will never be alone and powerless, as he was in the past, witnessing the defeat of medicine in the silence of the mortuary. The interdisciplinary approach to diagnosis, therapy, and prevention of cardiovascular diseases will place the cardiovascular pathologist working in a team with the cardiologist and cardiac surgeon on the one hand and the geneticist and molecular biologist on the other. Today, a wide spectrum of diagnostic tools is at the disposal of the pathologist, and some of them avoid the traditional microscope. They make our jobs more useful, and the cardiovascular pathologist's career can be intellectually and professionally appealing to the young generation of doctors to become protagonists in the fight against heart disease. The strategic position of cardiovascular pathologist, between the bench and the bedside, allows him or her to play a significant role in the study of cardiovascular disease.

It is time for cardiovascular pathology to move on. ${ }^{2,3}$ The extraordinary technologic breakthroughs in molecular biology, including microarrays and proteomics, provide exciting opportunities for investigating the causes besides the substrates of the diseases.

\section{References}

1. Titus JL, Daugherty GW, Kirklin JW, Edwards JE. Lesions of the atrioventricular conduction system after repair of ventricular septal defect. Relation to the heart block. Circulation. 1963;28:82-8.

2. Thiene G. Cardiovascular pathology: past, present and future achievements. Cardiovasc Pathol. 1992;1:79-86.

3. Billingham ME. Cardiovascular pathology at the crossroads: the dilemma of cardiac pathologists. Cardiovasc Pathol. 1992;1:3-5.

4. Barratt-Boyes BG, Simpson MM, Neutze JM. Intracardiac surgery in neonates and infants using deep hypothermia. Circulation. 1970;6162(suppl):III73.

5. Van Praagh R. The segmental approach to diagnosis in congenital heart disease. In: Birth defects, original articles series 8, 4. Baltimore: Williams \& Wilkins; 1972.

6. Milano A, Bortolotti U, Talenti E, et al. Calcific degeneration as the main cause of porcine bioprosthetic valve failure. Am J Cardiol. 1984;53:1066-70

7. Schoen FJ. Interventional and surgical cardiovascular pathology: clinical correlations and basic principles. Philadelphia: Saunders; 1989.

8. Fowles RE. Cardiac biopsy. Mount Kisco (NY): Futura Publishing Co Inc; 1992.

9. Richardson P, McKenna WJ, Bristow M, et al. Report of the 1995 WHO/ISFC Task Force on the definition and classification of cardiomyopathies. Circulation. 1996;93:841-2.

10. Thiene G, Nava A, Corrado D, Rossi L, Pennelli N. Right ventricular cardiomiopathy and sudden death in young people. $N$ Engl $\mathrm{J} \mathrm{Med}$. 1988;318:129-33

11. Tiso N, Stephan DA, Nava A, et al. Identification of mutations in the cardiac ryanodine receptor gene in families affected with arrhythmogenic right ventricular cardiomyopathy type 2 (ARVD2). Hum Mol Genet. 2001;10:189-194. 